\title{
The many ways COVID-19 affects households: consumption, time, and health outcomes
}

\section{George Davis ${ }^{1}$}

Accepted: 7 April 2021 / Published online: 15 April 2021

(C) The Author(s), under exclusive licence to Springer Science+Business Media, LLC, part of Springer Nature 2021

\begin{abstract}
Many of the direct and indirect effects of the COVID-19 pandemic are mediated mainly through the economics of the household. The Review of Economics of the Household has published 17 papers on interactions between COVID-19, ensuing policy responses, and the economics of the household. Five were published in the December 2020 issue, six in the March issue, and six in this June 2021 issue. Here I give an overview of 10 of these articles. The general conclusion is that consumption, time allocations, and health outcomes have all been significantly affected by the pandemic and ensuing policy responses, but the responses have been rather heterogenous across regions, individuals, and outcomes.
\end{abstract}

\section{Introduction}

To say the COVID-19 pandemic has been an unprecedented event now seems trite. Markets were decimated, unemployment soared, incomes evaporated, relationships strained, political differences exacerbated, and stress and uncertainty levels across the globe all increased. Most importantly, as of April 5, 2021 worldwide 2.85 million people had died from the pandemic (Johns Hopkins, Coronavirus Resource Center). While the impact of the pandemic and ensuing responses have been uneven across the globe, a common element exists: the pandemic and all its direct and indirect effects are mediated mainly through individuals making decisions within households. This implies that studies of the effects of the pandemic can often be considered economics of the household as broadly defined by this journal. Consequently, the Review of Economics of the Household called for papers to be submitted for a special issue on COVID-19. After the submissions, the decision was made to have the articles span several issues. A total of 17 articles were published, with five in

George Davis

georgedavis@vt.edu

1 Faculty of Health Sciences, Dept. Ag. \& Appl. Econ., Dept. Human Nutrition, Foods, and Exercise, Virginia Tech University, Blacksburg, VA, USA 
December 2020, six in March 2021, and six in this June 2021 issue. This essay serves as an introduction and overview of four from the December issue and all from this issue.

The household economics framework is very appropriate for analyzing how exogenous factors, such as the COVID-19 pandemic and ensuing policy responses, will affect household-related decision-making, activities, input choices, and outcomes, ranging from consumption patterns to time allocation to health outcomes. Importantly, because of its focus on intrahousehold resource allocations, household economics is also a natural framework for exploring inequalities, especially by gender.

The seven articles discussed in Section 2 below focus on consumption, time allocations, and labor market responses to some aspect of COVID-19. The three articles in Section 3 focus on the effectiveness of lockdown measures implemented to mitigate the spread of the virus.

\section{Consumption, time allocations, and gender inequalities in labor market effects}

In "The effect of COVID-19 lockdown and subsequent reopening on consumption in Iran" Mohammad Hoseini and Abolmohsen Valizadeh look at changes in consumption during the pandemic in Iran. They use monthly data on all purchases from the periods November-December 2018 to May-June 2020. The data covers 31 provinces and about 280 activities. The activities are then grouped into 12 aggregate groups: food \& beverages, tobacco, clothing \& footwear, housing repair \& expenses, appliance \& furniture, health, transport, communication, recreation, education, restaurants \& hotels, and miscellaneous goods and services. These are further aggregated into three categories: non-durables, semi-durables, and durables. An interesting component of their analysis is that they have both online and point-of-sales (POS) transactions. Using a difference-in-difference approach they compare sales in the November-December 2018 to June-July 2019 period, prior to COVID, with the same period in 2019 and 2020, after COVID started. With this framework they can look at heterogeneity across regions, but also across the three aggregate categories and the 12 sub-aggregate categories. Here I focus on the general findings. They find a $41 \%$ reduction in year-over-year growth rate of transactions associated with the strictest lockdown period. However, once the restrictions were relaxed, the yearover-year growth rate of transactions rises above the pre-pandemic time by about $12 \%$. Not surprisingly, they find that the tourism sector was hit the hardest, along with semi-durables like clothing. Non-durables, that would include food and beverages, were affected the least. Interestingly they find the pandemic led to a persistent switch to online shopping that did not fade away after the relaxation of restrictions, suggesting the pandemic may have revealed the convenience of online shopping to those who did not see it beforehand.

In a similar vein, Raymundo Campos-Vazques and Gerardo Esquivel look at consumption and geographic mobility in pandemic times based on evidence from Mexico. They use public data from the Mexican central bank on all point-of-sale (POS) transactions in the Mexican territory between January 1, 2019 and September 
30, 2020. Campos-Vazques and Esquivel analyze 12 aggregate expenditure categories of transactions: tourism, education, health care, food services, trade, transportation, insurance, telecommunications, supermarkets, big-box stores, and others. They find expenditures from April 1-September 2019 to April 1-September 2020 increased for four (healthcare, insurance, telecommunications, and other) but declined in the other eight categories, with especially large declines in tourism (70\%), food service (61\%), and transportation (66\%). As with most countries various lockdown measures were implemented over time in Mexico. To proxy the effect of the lockdown measures they create a mobility index and hypothesize the relationship between mobility and consumption is stronger in developing countries than developed countries. The underlying economic logic here is that in a less developed country like Mexico, in-person sales and therefore mobility may matter much more than in developed economies. Thus, mobility restrictions in such an economy are likely to have a larger impact on consumption levels. To test this hypothesis, they estimate a POS-mobility elasticity. In developed countries, estimates of this elasticity have been rather inelastic. In contrast, Campos-Vazques and Esquivel estimate a POS-mobility elasticity for Mexico close to unity. This supports their prediction that mobility restrictions will have larger impacts on consumption in less developed countries.

In addition to consumption patterns being affected by COVID-19, time allocation decisions have been affected significantly. Even prior to the pandemic, a general trend in labor markets has been the increase in teleworking or working from home. Based on data from the BLS (BLS Economic News Release, 2019), as of 2017-2018, $25 \%$ of wage and salary workers worked from home (WFH) occasionally and $15 \%$ had days where they worked exclusively from home. Furthermore, 57\% of workers had a flexible schedule allowing them to work from home at times. This implies one way to mitigate the labor market effects of COVID-19 would be to work from home if possible. Working from home frees up time normally devoted to commuting. Also simply by proximity, it allows for more time in household activities during breaks from formal work or multitasking.

In "The effect of working from home on major time allocations with a focus on food-related activities" Restrepo \& Zeballos (2020) use data on prime working-age adults (age 25-54 years old) who participated in the 2017-18 Leave and Job Flexibilities Module of the American Time Use Survey to look at differences in time allocated to three aggregate categories by individuals working at home and away from home. The three aggregate categories, and their subcategories are: labor market work, leisure-related time use (personal care, leisure, and sleeping), and food-related time use (food production and eating and drinking at home). Using a seemingly unrelated regression framework with a categorical dummy accounting for working from home and controlling for several individual characteristics, geographical and temporal indicators they find that those working from home spent less time working and on personal care, but more time on leisure, sleeping, and on food production and consumption than those who worked away from home. Regarding food at home, they find that those who worked at home with a partner present spent 25 more minutes engaged in food production and 48 more minutes eating and drinking at home than did individuals who worked away from home. These differences are quite substantial given that the sample average in terms of time spent in food production and eating 
and drinking are 33 and 31 min per day, respectively. Part of the focus on foodrelated activities is that research suggest that food at home is in general healthier than food away from home (Davis, 2014). Though this paper does not involve any type of COVID-19 simulations or analysis, the implications are rather clear: the closer the time use pattern of people who work from home because of COVID-19 are to those in this analysis, the more time in at-home food production may actually increase diet quality.

Though Restrepo and Zeballos do not provide any direct evidence of the relationship between time in home food production and diet quality, that is exactly one of the issues considered by Benjamin Scharadin, Yang Yu, Edward C. Jaenicke in their article "Household time activities, food waste, and diet quality: the impact of nonmarginal time changes due to COVID-19." As the title suggests, they are not only interested in how time allocations may change, but also how that in turn may affect diet quality and food waste. The significant amount of food waste in the world has become an important topic over the last decade, especially during COVID-19, as there is a simultaneous and significant amount of food waste and food insecurity in the world (e.g., Roe et al., 2021). Utilizing data from the American Time Use Survey (ATUS) in conjunction with data from the Household Food Acquisition and Purchase Survey (FoodAPS) during the period 2010-214, they model time allocations, food waste, and diet quality simultaneously. A novel aspect of their research is that they consider different food-related time activities separately (e.g., shopping versus preparation). They find that grocery store and food at home activities are associated with higher diet quality and lower food waste. However, time spent in secondary childcare and work time are negatively related to both diet quality and food waste. Furthermore, commuting and other household activities are only related to food waste. They use their model to simulate three COVID-19 scenarios: (i) the household head can work remotely, (ii) the household head lost their job, and (iii) the household head is considered an essential worker. They predict that in scenario (i), and for a household without children, there will be lower levels of food waste and higher diet quality. However, households without children in the other two COVID-19 scenarios are predicted to have only minor differences.

A basic question that arises anytime there is a reallocation of time is how it affects life satisfaction. While it is certainly the case that COVID-19 disrupted previous time schedules it should not immediately be concluded that life satisfaction decreased because of the time reallocation, ceteris paribus. In his December article, "Life satisfaction, loneliness and togetherness, with an application to COVID-19 lock downs" Hamermesh (2020) addresses this question from the following insightful perspective: when it comes to life satisfaction, what matters is not only what you do, but also who you do it with. Hamermesh uses the data from the 2012-2013 American Time Use Survey (ATUS) and the accompanying well-being module supplement for conducting the analysis. In the time diary, the ATUS not only asks what you were doing but who you were with while engaged in the activity. Satisfaction is measured in the analysis by the individual's ranking of their life satisfaction on a 10 (highest, "best possible life") to 0 (lowest, "worst possible life") scale.

Hamermesh finds that for married individuals, life satisfaction increases most with additional time spent with one's spouse. However, for singles satisfaction decreases as more time is spent alone. Satisfaction tends to decline with time spent sleeping or 
watching TV but increases with longer usual workweeks and higher income. Hamermesh uses his model to simulate three different time increases and income reduction scenarios that could occur with a COVID lockdown: (i) no loss of work or income, (ii) 1/3 of work time lost is spent watching television, but no loss of income, and (iii) scenario (ii) plus a $1 / 3$ loss of income. These three scenarios capture increasingly negative effects of a lock-down. The general finding is that for married couples, spending time with the spouse tends to mitigate the negative effects of the lack of freedom and decline in income. This is not the case for singles: additional time spent alone exacerbates low levels of generalized life satisfaction. Hamermesh also uses 2014-2015 British Time Use Survey and finds very similar results. The general interpretative hypothesis Hamermesh provides is that when we are free to choose, we spend time with those we prefer to spend time with. For many, work does not provide one with the freedom to choose who they spend time with but for married couples a lockdown allows them to spend more time with a preferred individual. Alternatively, for singles, spending time with work colleagues may be preferable to spending time alone. This general hypothesis is certainly very intuitive and makes sense, but it should be recognized it is NOT invalidated by the opposite result as well. The hypothesis is also completely consistent with some individuals preferring to spend more time at work than with partners. This is only brought up as several papers have found that domestic violence increased during the pandemic (e.g., Hsu \& Henke, 2021) and it is hard to imagine in those cases that the abused individual would prefer to spend time at home with the abuser rather than with colleagues at work (i.e., ceteris paribus matters).

As Croda \& Grossbard (2021) summarized in their introduction to the March issue on COVID, and as demonstrated in those articles, the impact of COVID has been unequal across genders. The rather consistent finding across multiple studies is that women have paid a much grater price with respect to COVID than men. Sandeep Mohapatra's article "Gender differentiated economic responses to crisis in developing countries: insights for COVID-19 recovery policies" adds to these general findings. Mohapatra constructs country-level panel datasets from 1991-2019 for two regions: South Asia and West Africa. A series of panel autoregressive models are estimated for each of the outcome variables: employment by gender and household consumption, for South Asia and West Africa, and access to sanitation for West Africa. A qth order lag operator is applied to a dummy variable that is created for each year in which there was a negative growth in GDP per capita. Focusing here on the employment effects, for South Asia the results strongly support the hypothesis that the effect of an economic growth shock for women's work is negative and persistent over time without signs of recovery. The loss of employment is estimated to be more than double that of men within five years after a shock. Male unemployment is much more transient and dissipates over time. In West Africa, female employment increases immediately after the shock as perhaps a coping mechanism to help offset lost household income. However, this surge disappears over time and unemployment persists. This study provides insights that can help design and target COVID-19 recovery policies.

An interesting issue related to both unemployment and COVID-19 is the extent to which individuals may be able to mitigate unemployment risk by having different work from home options. Cecilia Peluffo and Mariana Viollaz consider the 
intrahousehold differences in exposure to labor market shocks in their article "Intrahousehold exposure to labor market risk in the time of Covid-19: lessons from Mexico." Peluffo and Viollaz pull together data from several sources over the time period 2015-2020 and create a working from home index (WFH) for each individual in the household. The index is based on job characteristic tasks and internet connectivity in the home. Armed with this index they consider four main issues: (i) how the index varies across demographic and employment profiles, (ii) how the index is related to aggregate changes in formal employment (iii) what is the intrahousehold correlation between the indexes of spouses and (iv) the correlation between the index and access to formal credit and informal consumption smoothing. One of the most interesting findings is that women have a larger average WFH index than men. Peluffo and Viollaz attribute this to women working in professions that are less physically demanding and require less face-to-face interactions. They find that working in occupations with a low WFH index value is associated with the lower tail of the income distribution, being employed in small-size firms, and not owning your own home. In addition, the WFH index tends to be lower when social distancing policies are in place. There is also a high positive correlation between spouses' WFH indexes which limits the household's ability to hedge against unemployment in an intrahousehold risk sharing context. Finally, low WFH indexes are associated with less access to formal credit. All this suggests that existing income inequalities are likely exacerbated during the pandemic.

\section{The effectiveness of lockdown measures: behavior, infection, and fatalities}

Around the world, in an attempt to slow the spread of COVID-19, governments responded with a variety of lockdown measures, such as curfews, stay-at-home orders, commercial and non-commercial assembly size restrictions, school closings, social distancing, travel restrictions, quarantines, and mask wearing requirements. A challenging aspect of many of the lockdown measures is their voluntary nature. Even for those that are not voluntary, enforcement can be difficult. Thus, lockdown measures imply tension between negative consequences of reduced economic and social activity and positive health effects of reducing the spread of the disease. As a result, the implementation, timing, acceptance, and effectiveness of lockdown measures has been quite heterogeneous and contentious across the globe.

Given that many of the lockdown measures were and are voluntary, and not abiding by them can be considered risky behavior, a natural economic question that arises is: what factors determine the decision to abide by the lockdown guidelines? Sheth \& Wright (2020) address this question by considering three main classical economic determinants in "The usual suspects: do risk tolerance, altruism, and health predict the response to COVID-19?" Six days after the California stay-at-home orders went into effect, Sheth and Wright administered two survey instruments to 338 undergraduate economics students at a large California university between March 26th and April 7th, 2020. The surveys included questions about adhering to stay-at-home orders, altruism, risk preferences, likely exposure to COVID-19, beliefs about the effectiveness of social distancing, and several demographic variables. 
Sheth and Wright found that $25 \%$ of the students violated the stay-at-home order. However, none of the classical determinants, risk preferences, altruism, or preexisting health conditions, were predictive of compliance with the stay-at-home order. Sheth and Wright conclude these results raise doubts that minimally enforced social distancing policies will be very effective, at least for this age cohort. From a purely explanatory standpoint, clearly there are other factors that explain the lack of compliance and this is where complementary insights from behavioral economics could play a role. Risky behaviors that have a delayed effect are often studied in the context of an intertemporal choice problem and it is well established in that literature, especially for youth, that there is an inherent present consumption bias: now is preferred to later (e.g., Frederick et al., 2002). Coupling this with the uncertainty associated with contracting the disease and the severity of the disease, if contracted, further pushes the individual toward a present consumption bias. In addition, there are well-known information processing or cognitive biases, such as confirmation bias and overconfidence bias (e.g., Pliske \& Mutter, 1996; Jones \& Sugden, 2001), that would tend to undermine the effectiveness of lockdown policies as well, especially for younger cohorts.

It is well established that the elderly are more susceptible to contracting and having more severe effects from COVID-19 than the young, leading to an obvious public health concern: to what extent is there cross-infection from young to old? The answer to this question is extremely important because if the cross-infection rate is low, then this would allow for more targeted age-dependent relaxation of lockdown measures (e.g., opening of schools) without much concern for an increase risk to the more vulnerable older population. This is the focus of Harris's (2020) article, "Data from the COVID-19 epidemic in Florida suggest that younger cohorts have been transmitting their infections to less socially mobile older adults." Using data from Florida's sixteen most populous counties collected between March 1, 2020 and June 27, 2020, Harris analyzes the daily incidence of newly reported COVID-19 cases among adults aged 20-39 years, 40-59 years, and 60 or more years. For all three age groups in all 16 counties, the reported COVID-19 case incidences increased soon after the governor-ordered Full Phase 1 reopening went into effect. As part of this analysis, Harris investigates the often-heard absurd casual claim that cases were higher because of more testing. Harris finds that hospitalization data does not support this conjecture. The most important finding is that the younger cohort first acquired the infection through increased social contact with their peers and then transmitted their infections to older, less socially mobile individuals. The conclusion then is that there would be no isolated sustainable epidemic among older people without cross-infection from younger individuals. Stated more simply: in transmission the virus does not care if you are young or old and thus age-targeted lockdowns are likely less effective than comprehensive lockdowns. This result, coupled with that of Sheth and Wright, underscores the importance of getting younger individuals to comply with lockdown measures.

The most pressing and obvious question of interest is to what extent the timing of different lock-down measures affected fatalities and this is the question addressed by Ainoa Aparicio and Shoshana Grossbard in "Are COVID fatalities in the US higher than in the EU, and if so why?" This article is a great example of the importance of distinguishing between unconditional and conditional differences in means. More specifically, a comparison of unconditional means does not provide any insights into 
to underlying differences in covariates, whereas a conditional mean will take into account those differences and thus help explain why there are differences or, once the covariate differences are taken into account, why there are no real differences. The approach taken by Aparicio and Grossbard is to estimate log-linear regressions of the $\log$ of cumulative number of deaths post 100 days since the pandemic's onset using a sample of 85 nations/states: 35 European countries and 50 US states using data from various sources and years 2010, 2017, 2018, 2020. They begin by running a model that just controls for population and find that the US/Europe gap in cumulative deaths is $130 \%$ and statistically significant. The focus is then to establish what happens to this gap as more covariates are added. When demographic and some economic factors are added, the US/Europe gap shrinks to $100 \%$ but is still statistically significant. Finally, and most importantly, once differences in social distance implementation and timing are added to the model, the US/Europe gap shrinks further and becomes statistically insignificant. This is a rather intuitive result in that European countries were more likely to implement social distancing at a faster pace than the US. This finding is consistent with other research indicating the speed of responding plays a crucial role in curtailing the spread of the disease (Ngonghala et al., 2020). The inference then is that the main reason for the difference in the unconditional means is due to the differences in all these factors, but especially the differences in the timing of social distancing measures.

\section{Conclusion}

As the articles in this overview demonstrate, the COVID-19 pandemic can impact a broad range of topics within the economics of the household. The topics covered here range from consumption changes to time allocation changes to health outcome effects. The articles covered countries from around the globe. The overarching conclusions adhere with casual empiricism: the effects of COVID-19 have been very significant and the mitigation policies, and their effectiveness, have been very heterogeneous.

Acknowledgements I thank Shoshana Grossbard for helpful comments and suggestions.

\section{Compliance with ethical standards}

Conflict of interest The author declares no competing interests.

Publisher's note Springer Nature remains neutral with regard to jurisdictional claims in published maps and institutional affiliations.

\section{References}

Bureau of Labor Statistics. Economic news release. Job flexibilities and work schedules. 2019. https:// www.bls.gov/news.release/flex2.nr0.htm.

Croda, E., \& Grossbard, S. (2021). Women pay the price of COVID-19 more than men. Review of Economics of the Household, 19(1), 1-9. 
Davis, G. (2014). Food at home production and consumption: implications for nutrition quality and policy. Review of Economics of the Household, 12(3), 565-588.

Frederick, S., Loewenstein, G., \& O'Donoghue, T. (2002). Time discounting and time preference: a critical review. Journal of Economic Literature, 40(2), 351-401.

Harris, J. (2020). Data from the COVID-19 epidemic in Florida suggest that younger cohorts have been transmitting their infections to less socially mobile older adults. Review of Economics of the Household, 18(4), 1019-1037.

Hamermesh, D. (2020). Life satisfaction, loneliness and togetherness, with an application to Covid-19 lock-downs. Review of Economics of the Household, 18(4), 983-1000.

Hsu, L., \& Henke, A. (2021). COVID-19, staying at home, and domestic violence. Review of Economics of the Household, 19(1), 145-155

John's Hopkins University. Coronavirus Resource Center. https://coronavirus.jhu.edu/.

Jones, M., \& Sugden, R. (2001). Positive confirmation bias in the acquisition of information. Theory and Decision, 50(1), 59-99.

Ngonghala, C., Enahoro, A., \& Gumel, A. (2020). Could masks curtail the post-lockdown resurgence of COVID-19 in the US? Mathematical Biosciences, 329, 108452.

Pliske, R., \& Mutter, S. (1996). Age differences in the accuracy of confidence judgments. Experimental Aging Research, 22(2), 199-216.

Restrepo, B., \& Zeballos, E. (2020). The effect of working from home on major time allocations with a focus on food-related activities. Review of Economics of the Household, 18(4), 1165-1187.

Roe, B., Bender, K., \& Qi, D. (2021). The impact of COVID-19 on consumer food waste. Applied Economic Perspectives and Policy, 43(1), 401-411.

Sheth, K., \& Wright, G. (2020). The usual suspects: do risk tolerance, altruism, and health predict the response to COVID-19? Review of Economics of the Household, 18(4), 1041-1052. 